\title{
Learning genetic epistasis using Bayesian network scoring criteria
}

Xia Jiang ${ }^{1 *}$, Richard E Neapolitan ${ }^{5}$, M Michael Barmada $^{4}$ and Shyam Visweswaran ${ }^{1,2,3}$

\begin{abstract}
Background: Gene-gene epistatic interactions likely play an important role in the genetic basis of many common diseases. Recently, machine-learning and data mining methods have been developed for learning epistatic relationships from data. A well-known combinatorial method that has been successfully applied for detecting epistasis is Multifactor Dimensionality Reduction (MDR). Jiang et al. created a combinatorial epistasis learning method called BNMBL to learn Bayesian network (BN) epistatic models. They compared BNMBL to MDR using simulated data sets. Each of these data sets was generated from a model that associates two SNPs with a disease and includes 18 unrelated SNPS. For each data set, BNMBL and MDR were used to score all 2-SNP models, and BNMBL learned significantly more correct models. In real data sets, we ordinarily do not know the number of SNPs that influence phenotype. BNMBL may not perform as well if we also scored models containing more than two SNPs. Furthermore, a number of other BN scoring criteria have been developed. They may detect epistatic interactions even better than BNMBL.

Although BNs are a promising tool for learning epistatic relationships from data, we cannot confidently use them in this domain until we determine which scoring criteria work best or even well when we try learning the correct model without knowledge of the number of SNPs in that model.
\end{abstract}

Results: We evaluated the performance of $22 \mathrm{BN}$ scoring criteria using 28,000 simulated data sets and a real Alzheimer's GWAS data set. Our results were surprising in that the Bayesian scoring criterion with large values of a hyperparameter called $\alpha$ performed best. This score performed better than other BN scoring criteria and MDR at recall using simulated data sets, at detecting the hardest-to-detect models using simulated data sets, and at substantiating previous results using the real Alzheimer's data set.

Conclusions: We conclude that representing epistatic interactions using BN models and scoring them using a BN scoring criterion holds promise for identifying epistatic genetic variants in data. In particular, the Bayesian scoring criterion with large values of a hyperparameter $\alpha$ appears more promising than a number of alternatives.

\section{Background}

The advent of high-throughput genotyping technology has brought the promise of identifying genetic variations that underlie common diseases such as hypertension, diabetes mellitus, cancer and Alzheimer's disease. However, our knowledge of the genetic architecture of common diseases remains limited; this is in part due to the complex relationship between the genotype and the phenotype. One likely reason for this complex relationship arises from gene-gene and gene-environment interactions. So an

\footnotetext{
*Correspondence: xij6@pitt.edu

'Department of Biomedical Informatics, University of Pittsburgh, Pittsburgh, PA, USA

Full list of author information is available at the end of the article
}

important challenge in the analysis of high-throughput genetic data is the development of computational and statistical methods to identify gene-gene interactions. In this paper we apply Bayesian network scoring criteria to identifying gene-gene interactions from genome-wide association study (GWAS) data.

As background we review gene-gene interactions, GWAS, Bayesian networks, and modeling gene-gene interactions using Bayesian networks.

\section{Epistasis}

In Mendelian diseases, a genetic variant at a single locus may give rise to the disease [1]. However, in many common diseases, it is likely that manifestation of the
C Biomed Central

(c) 2011 Jiang et al; licensee BioMed Central Ltd. This is an Open Access article distributed under the terms of the Creative Commons Attribution License (http://creativecommons.org/licenses/by/2.0), which permits unrestricted use, distribution, and reproduction in any medium, provided the original work is properly cited. 
disease is due to genetic variants at multiple loci, with each locus conferring modest risk of developing the disease. For example, there is evidence that gene-gene interactions may play an important role in the genetic basis of hypertension [2], sporadic breast cancer [3], and other common diseases [4]. The interaction between two or more genes to affect a phenotype such as disease susceptibility is called epistasis. Biologically, epistasis likely arises from physical interactions occurring at the molecular level. Statistically, epistasis refers to an interaction between multiple loci such that the net affect on phenotype cannot be predicted by simply combining the effects of the individual loci. Often, the individual loci exhibit weak marginal effects; sometimes they may exhibit none.

The ability to identify epistasis from genomic data is important in understanding the inheritance of many common diseases. For example, studying genetic interactions in cancer is essential to further our understanding of cancer mechanisms at the genetic level. It is known that cancerous cells often develop due to mutations at multiple loci, whose joint biological effects lead to uncontrolled growth. But many cancer-associated mutations and interactions among the mutated loci remain unknown. For example, highly penetrant cancer susceptibility genes, such as BRCA1 and BRCA2, are linked to breast cancer [5]. However, only about 5 to 10 percent of breast cancer can be explained by germ-line mutations in these single genes. "Most women with a family history of breast cancer do not carry germ-line mutations in the single highly penetrant cancer susceptibility genes, yet familial clusters continue to appear with each new generation" [6]. This kind of phenomenon is not yet well understood, and undiscovered mutations or undiscovered interactions among mutations are likely responsible.

Recently, machine-learning and data mining techniques have been developed to identify epistatic interactions in genomic data. Such methods include combinatorial methods, set association analysis, genetic programming, neural networks and random forests [7]. A well-known combinatorial method is Multifactor Dimensionality Reduction (MDR) [3,8-10]. MDR combines two or more variables into a single variable (hence leading to dimensionality reduction); this changes the representation space of the data and facilitates the detection of nonlinear interactions among the variables. MDR has been successfully applied to detect epistatic interactions in diseases such as sporadic breast cancer [3] and type II diabetes [8], typically in data sets containing at most a few hundred genetic loci.

\section{GWAS}

The most common genetic variation is the single nucleotide polymorphism (SNP) that results when a single nucleotide is replaced by another in the genomic sequence. In most cases a SNP is biallelic, that is it has only two possible values among $A$ and $G$ or $C$ and $T$ (the four DNA nucleotide bases). If the alleles are $A$ and $G$, a diploid individual has the SNP genotype $A A$, $G G$, or $A G$. The less frequent (rare) allele must be present in $1 \%$ or more of the population for a site to qualify as a SNP [11]. The human genome contains many millions of SNPs. In what follows we will refer to SNPs as the loci investigated when searching for a correlation of some loci with a phenotype such as disease susceptibility.

The advent of high-throughput technologies has enabled genome-wide association studies (GWAS). A GWAS involves sampling in a population of individuals about 500,000 representative SNPs. Such studies provide researchers unprecedented opportunities to investigate the complex genetic basis of diseases. While the data in a GWAS have commonly been analyzed by investigating the association of each locus individually with the disease [12-16], there has been application of pathway analysis in some of these studies $[15,16]$.

An important challenge in the analysis of genome-wide data sets is the identification of epistatic loci that interact in their association with disease. Many existing methods for epistasis learning such as combinatorial methods cannot handle a high-dimensional GWAS data set. For example, if we only investigated all 0, 1, 2, 3 and 4-SNP combinations when there are 500,000 SNPs, we would need to investigate $2.604 \times 10^{21}$ combinations. Researchers are just beginning to develop new approaches for learning epistatic interactions using a GWAS data set [17-24]; however, the successful analysis of epistasis using high-dimensional data sets remains an open and vital problem. Cordell [25] provides a survey of methods currently used to detect gene-gene interactions that contribute to human genetic diseases. Most GWAS studies so far have been about "agnostic" discovery. Thomas [26] suggests combining data-driven approaches with hypothesis-driven, pathway-based analysis using hierarchical modeling strategies.

\section{Bayesian Networks}

Bayesian networks [27-33] are increasingly being used for modeling and knowledge discovery in genetics and in genomics [34-41]. A Bayesian network (BN) is a probabilistic model that consists of a directed acyclic graph (DAG) G, whose nodes represent random variables, and a joint probability distribution $P$ that satisfies the Markov condition with $G$. We say that $(G, P)$ satisfies the Markov condition if each node (variable) in $G$ is conditionally independent of the set of all its nondescendent nodes in $G$ given the set of all its parent nodes. It is a theorem [31] that $(G, P)$ satisfies the Markov condition 
(and therefore is a $\mathrm{BN}$ ) if and only if $P$ is equal to the product of the conditional distributions of all nodes given their parents in $G$, whenever these conditional distributions exist. That is, if the set of nodes is $\left\{X_{1}, X_{2}, \ldots\right.$, $\left.X_{n}\right\}$, and $P A_{i}$ is the set of parent nodes of $X_{1}$, then

$$
P\left(X_{1}, X_{2}, \ldots, X_{n}\right)=\prod_{i=1}^{n} P\left(X_{i} \mid P A_{i}\right) .
$$

BNs are often developed by first specifying a DAG that satisfies the Markov condition relative to our belief about the probability distribution, and then determining the conditional distributions for this DAG. One common way to specify the edges in the DAG is to include the edge $X_{1} \rightarrow X_{2}$ only if $X_{1}$ is a direct cause of $X_{2}$ [32]. Figure 1 shows an example of a BN. A BN can be used to compute conditional probabilities of interest using a $\mathrm{BN}$ inference algorithm [32]. For example, we can compute the conditional probability that an individual has lung cancer and the conditional probability the individual has bronchitis given that the individual has a history of smoking and a positive chest $X$-ray.

Both the parameters and the structure of a $\mathrm{BN}$ can be learned from data. The Data consists of samples from some population, where each sample (called a data item) is a vector of values for all the random variables under consideration. Learning the structure of a $\mathrm{BN}$ is more challenging than learning the parameters of a specified BN structure, and a variety of techniques have been developed for structure learning. One method for structure learning, called constraint-based, employs statistical tests to identify DAG models that are consistent

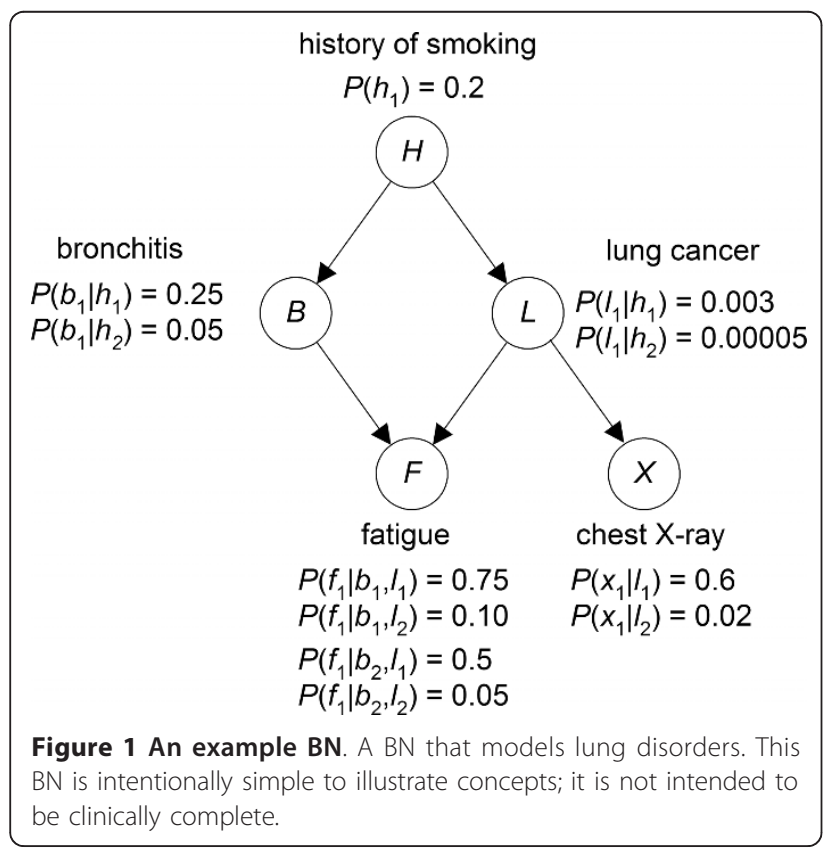

with the conditional independencies entailed by the data [42]. A second method, called score-based, employs heuristic search to find DAG models that maximize a desired scoring criterion [32]. Pierrier et al. [43] provide a detailed review of the methods for $\mathrm{BN}$ structure learning. Next we review scoring criteria since these criteria are the focus of this paper.

\section{BN Scoring Criteria}

We review several BN scoring criteria for scoring DAG models in the case where all variables are discrete since this is the case for the application we will consider. BN scoring criteria can be broadly divided into Bayesian and information-theoretic scoring criteria.

\section{Bayesian scoring criteria}

The Bayesian scoring criteria compute the posterior probability distribution, starting from a prior probability distribution on the possible DAG models, conditional on the Data. For a DAG G containing a set of discrete random variables $V=\left\{X_{1}, X_{2}, \ldots, X_{n}\right\}$ and Data, the following Bayesian scoring criterion (or simply score) is derived under the assumption that all DAG models are equally likely a priori $[44,45]$ :

$$
\begin{aligned}
\text { score }_{\text {Bayes }}(G: \text { Data }) & =P(\text { Data } \mid G) \\
& =\prod_{i=1}^{n} \prod_{j=1}^{q_{i}} \frac{\Gamma\left(\sum_{k=1}^{r_{i}} a_{i j k}\right)}{\Gamma\left(\sum_{k=1}^{r_{i}} a_{i j k}+\sum_{k=1}^{r_{i}} s_{i j k}\right)} \prod_{k=1}^{r_{i}} \frac{\Gamma\left(a_{i j k}+s_{i j k}\right)}{\Gamma\left(a_{i j k}\right)},
\end{aligned}
$$

where $r_{i}$ is the number of states of $X_{i}, q_{i}$ is the number of different values the parents of $X_{i}$ in $G$ can jointly assume, $a_{i j k}$ is the prior belief concerning the number of times $X_{i}$ took its $k$ th value when the parents of $X_{i}$ took their $j$ th value, and $s_{i j k}$ is the number of times in the data that $X_{i}$ took its $k$ th value when the parents of $X_{i}$ took their $j$ th value.

The Bayesian score given by Equation 1 assumes that our prior belief concerning each unknown parameter in each DAG model is represented by a Dirichlet distribution, where the hyperparameters $a_{i j k}$ are the parameters for this distribution. Cooper and Herskovits [44] suggest setting the value of every hyperparameter $a_{i j k}$ equal to 1 , which assigns a prior uniform distribution to the value of each parameter (prior ignorance as to its value). Setting all hyperparameters to 1 yields the $K 2$ score and is given by the following equation:

$$
\operatorname{score}_{K 2}(G: \text { Data })=\prod_{i=1}^{n} \prod_{j=1}^{q_{i}} \frac{\Gamma\left(r_{i}\right)}{\Gamma\left(r_{i}+\sum_{k=1}^{r_{i}} s_{i j k}\right)} \prod_{k=1}^{r_{i}} \Gamma\left(1+s_{i j k}\right) \text {. }
$$

The K2 score does not necessarily assign the same score to Markov equivalent DAG models. Two DAGs are Markov equivalent if they entail the same conditional independencies. For example, the DAGs $X \rightarrow Y$ and $X \leftarrow$ $Y$ are Markov equivalent. Heckerman et al. [45] show that if we determine the values of the hyperparameters 
from a single parameter $\alpha$ called the prior equivalent sample size then Markov equivalent DAGs obtain the same score. If we use a prior equivalent sample size $\alpha$ and want to represent a prior uniform distribution for each variable (not parameter) in the network, then for all $i, j$, and $k$ we set $a_{i j k}=\alpha / r_{i} q_{i}$, where $r_{i}$ is the number of states of the $i$ th variable and $q_{i}$ is the number of different values the parents of $X_{i}$ can jointly assume. When we use a prior equivalent sample size $\alpha$ in the Bayesian score, the score is called the Bayesian Dirichlet equivalent (BDe) scoring criterion. When we also represent a prior uniform distribution for each variable, the score is called the Bayesian Dirichlet equivalent uniform (BDeu) scoring criterion and is given by the following equation:

$$
\operatorname{score}_{\alpha}(G: \text { Data })=\prod_{i=1}^{n} \prod_{j=1}^{q_{i}} \frac{\Gamma\left(\alpha / q_{i}\right)}{\Gamma\left(\alpha / q_{i}+\sum_{k=1}^{r_{i}} s_{i j k}\right)} \prod_{k=1}^{r_{i}} \frac{\Gamma\left(\alpha / r_{i} q_{i}+s_{i j k}\right)}{\Gamma\left(\alpha / r_{i} q_{i}\right)} .
$$

The Bayesian score does not explicitly include a $D A G$ penalty for network complexity. However, a DAG penalty is implicitly determined by the hyperparameters $a_{i j k}$. Silander et al. [46] show that if we use the BDeu score, then the DAG penalty decreases as $\alpha$ increases. The K2 score uses hyperparameters in a way that can be related to a prior equivalent sample size. When a node is modeled as having more parents, the $\mathrm{K} 2$ score effectively assigns a higher prior equivalent sample size to that node, which in turn decreases its DAG penalty.

\section{Minimum description length scoring criteria}

The Minimum Description Length (MDL) Principle is an information-theoretic principle [47] which states that the best model is one that minimizes the sum of the encoding lengths of the data and the model itself. To apply this principle to scoring DAG models, we must determine the number of bits needed to encode a DAG $G$ and the number of bits needed to encode the data given the DAG. Suzuki [48] developed the following $M D L$ scoring criterion:

$$
\text { scoresuz } \left._{(G}: \text { Data }\right)=\sum_{i=1}^{n} \frac{d_{i}}{2} \log _{2} m-m \sum_{i=1}^{n} \sum_{j=1}^{q_{i}} \sum_{k=1}^{r_{i}} P\left(x_{i k}, p a_{i j}\right) \log _{2} \frac{P\left(x_{i k}, p a_{i j}\right)}{P\left(x_{i k}\right) P\left(p a_{i j}\right)},
$$

where $n$ is the number of nodes in $G, d_{i}$ is the number of parameters needed to represent the conditional probability distributions associated with the $i$ th node in $G, m$ is the number of data items, $r_{i}$ is the number of states of $X_{i}, x_{i k}$ is the $k$ th state of $X_{i}, q_{i}$ is the number of different values the parents of $X_{i}$ can jointly assume, $p a_{i j}$ is the $j$ th value of the parents of $X_{i}$, and the probabilities are estimated from the Data. In Equation 2 the first sum is the DAG penalty, which is the number of bits sufficient to encode the DAG model, and the second term is the number of bits sufficient to encode the Data given the model.
Other MDL scores assign different DAG penalties and therefore differ in the first term in Equation 2, but encode the data the same. For example, the Akaike Information Criterion (AIC) score is an MDL scoring criterion that uses $\sum_{i=1}^{n} d_{i}$ as the DAG penalty. We will call this score score $_{A I C}$. In the DDAG Model section (acronym DDAG is defined in that section) we give an MDL score designed specifically for scoring BNs representing epistatic interactions.

\section{Minimum message length scoring criterion}

Another score based on information theory is the Minimum Message Length Score (MML) that is described in [30]. In the case of discrete variables it is equal to

$$
\text { score }_{M M L}(G: \text { Data })=\sum_{i=1}^{n} d_{i}\left(\log _{2} \frac{e^{3 / 2} \pi}{6}\right)-\log _{2} \operatorname{score}_{K 2}(G \quad: \quad \text { Data })
$$

where $d_{i}$ is the number of parameters stored for the $i$ th node in $G$ and score $_{k 2}$ is the K2 score mentioned previously.

To learn a DAG model from data, we can score all DAG models using one of the scores just discussed and then choose the highest scoring model. However, when the number of variables is not small, the number of candidate DAGs is forbiddingly large. Moreover, the $\mathrm{BN}$ structure learning problem has been shown to be NP-hard [49]. So heuristic algorithms have been developed to search over the space of DAGs during learning [32].

In the large sample limit, all the scoring criteria favor a model that most succinctly represents the generative distribution. However, for practical sized data sets, the results can be quite disparate. Silander et al. [46] provide a number of examples of learning models from various data sets showing that the choice of $\alpha$ in the BDeu scoring criterion can greatly affect how many edges exist in the selected model. For example, in the case of their Yeast data set (which contains 9 variables and 1484 data items), the number of edges in the selected model ranged from 0 to 36 as the value of $\alpha$ in the Bayesian scores ranged from $2 \times 10^{-20}$ to 34,000 . Although researchers have recommended various ways for choosing $\alpha$ and sometimes argued for the choice on philosophical/intuitive grounds [32], there is no agreed upon choice.

\section{Detecting Epistasis Using BNs}

BNs have been applied to learning epistatic interactions from GWAS data sets. Han et al. [50] developed a Markov blanket-based method that uses a $\mathrm{G}^{2}$ test instead of a BN scoring criterion. Verzilli et al. [51] represent the relationships among SNPs and a phenotype using a Markov network $(M N)$, which is similar to a $\mathrm{BN}$ but contains undirected edges. They then use MCMC to do 
approximate model averaging to learn whether a particular edge is present. Both these methods model the relationships among SNPs besides the relationship between SNPs and a phenotype.

Jiang et al. [52] took a different approach. Since we are only concerned with discovering SNP-phenotype relationships, they used specialized BNs called DDAGs to model these relationships. DDAGs are discussed in the DDAG Model subsection of the Results section. They developed a combinatorial epistasis learning method called BNMBL that uses an MDL scoring criterion for scoring DDAGs. They compared BNMBL to MDR using the data sets developed in [10]. Each of these data sets was generated from a model that associates two SNPs with a disease and includes 18 unrelated SNPs. For each data set, BNMBL and MDR were used to score all 2-SNP models, and BNMBL learned significantly more correct models. In another study, Visweswaran et al. [53] employed a K2-based scoring criterion for scoring these same DAG models that also outperformed MDR.

In real data sets, we ordinarily do not know the number of SNPs that influence phenotype. BNMBL may not perform as well if we also scored models containing more than two SNPs. Although BNs are a promising tool for learning epistatic relationships from data, we cannot confidently use them in this domain until we determine which scoring criteria work best or even well when we try learning the correct model without knowledge of the number of SNPs in that model. We provide results of experiments investigating this performance in the Results section.

\section{Diagnostic BNs Containing SNP Variables}

BN diagnostic systems that contain SNP information have also been learned from data. For example, Sebastiani et al. [54] learned a BN that predicts stroke in individuals with sickle cell anemia, while Meng et al. [55] learned a $\mathrm{BN}$ that predicts rheumatoid arthritis. In these studies candidate SNPs were identified based on known metabolic pathways. This is in contrast to the agnostic search ordinarily used to analyze GWAS data sets (discussed above). For example, Sebastiani et al. [54] identified 80 candidate genes and analyzed 108 SNPs in these genes.

\section{Results}

We first describe the BN model used to model SNP interactions associated with disease. Next, we develop a BN score tailored to this model and list the other $\mathrm{BN}$ scores that are evaluated. Finally, we provide the results of experiments that evaluate the various $B N$ scores and MDR using simulated data and a real GWAS data set.

\section{The DDAG Model}

We use BNs to model the relationships among SNPs and a phenotype such as disease susceptibility. Given a set of SNPs $\left\{S_{1}, S_{2}, \ldots, S_{n}\right\}$ and a disease $D$, we consider all DAGs in which node $D$ has only incoming edges and no outgoing edges. Such DAGs have the causal interpretation that SNPs are either direct or indirect causes of disease. An example of a DAG for 9 SNPs is shown in Figure 2. This DAG does not represent the relationships among gene expression levels. Rather it represents the statistical dependencies involving the disease status and the alleles of the SNPs. Since we are only concerned with modeling the dependence of the disease on the SNPs and not the relationships among the SNPs, there is no need for edges between SNPs. So we need only consider DAGs where the only edges are ones to $D$. An example of such a DAG is shown in Figure 3. We call such a model a direct $D A G(D D A G)$.

The number of DAGs that can be constructed is forbiddingly large when the number of nodes is not small. For example, there are $\sim 4.2 \times 10^{18}$ possible DAGs for a domain with ten variables [56]. The space of DDAGs is much smaller: there are $2^{n}$ DDAGs, where $n$ is the number of SNPs. So if we have ten SNPs, there are only $2^{10}$ DDAGs. Though the model space of DDAGs is much smaller that the space of DAGs, it still remains exponential in the number of variables. In the studies reported here, we search in the space of DDAGs.

\section{The BN Minimum Bit Length (BNMBL) Score}

An MDL score called BNMBL that is adapted to DDAGs is developed next. Each parameter (conditional probability) in a DAG model learned from data is a fraction with precision $1 / m$, where $m$ is the number of data items. Therefore, it requires $O\left(\log _{2} m\right)$ bits to store each parameter. However, as explained in [57], the high order bits are not very useful. So we need use

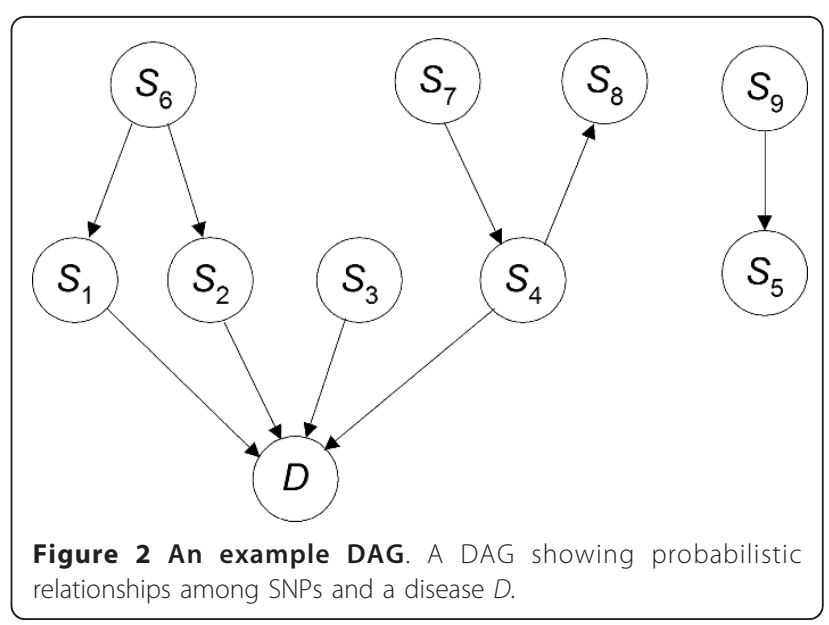




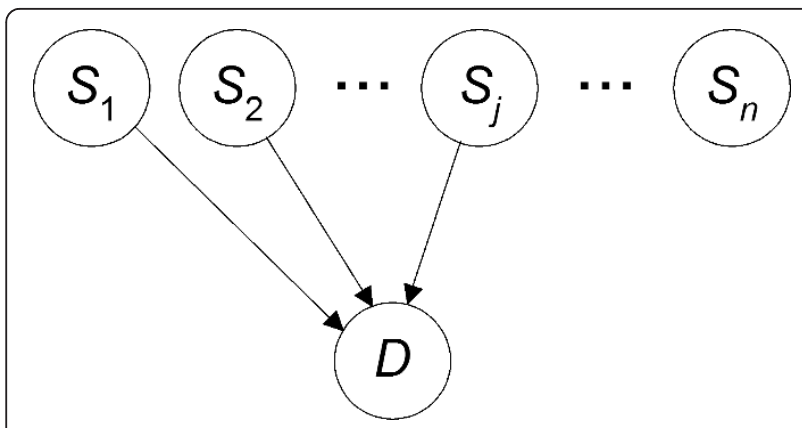

Figure 3 An example DDAG. A DDAG showing probabilistic relationships between SNPs and a disease D. A DDAG differs from the DAG in Figure 2 in that the relationships among the SNPs are not represented.

only $\frac{1}{2} \log _{2} m$ bits and we arrive at the DAG penalty in Equation 2.

Suppose that $k$ SNPs have edges into $D$ in a given DDAG. Since each SNP has three possible values, there are $3^{k}$ joint states of the parents of $D$. The expected value of the number of data items, whose values for these $k$ SNPs are the values in each joint state, is $m / 3^{k}$. If we approximate the precision for each of $D$ 's parameters by this average, the penalty for each of these parameters is $\frac{1}{2} \log _{2} \frac{m}{3^{k}}$. Since the penalty for each parameter in a parent SNP is $\frac{1}{2} \log _{2} m$, the total DAG penalty for a DDAG model is

$$
\frac{3^{k}}{2} \log _{2} \frac{m}{3^{k}}+\frac{2 k}{2} \log _{2} m
$$

The multiplier 2 appears in the second term because each SNP has three values. We need store only two of the three parameters corresponding to the SNP states, since the value of the remaining parameter is uniquely determined given the other two. No multiplier appears in the first term because the disease node has only two values. When we use this DAG penalty in an MDL score (Equation 2), we call the score score $_{E p i}$.

\section{BN Scoring Criteria Evaluated}

We evaluated the performance of MDR; three MDL scores: score $_{E p i}$, score $_{S u z}$, and score $_{A I C}$; two Bayesian scores: score $_{K 2}$, and score $_{\alpha}$; and the information-theoretic score score $_{M M L}$. For score $_{\alpha}$ we performed a sensitivity analysis over the following values of $\alpha=13,6,9,12,15$, $18,21,24,30,54,162$. We evaluated two versions of each of the MDL scores. In the first version, all $n$ SNPs in the domain are included in the model, though only $k$ of them directly influence $D$ and hence have edges to $D$ in the DDAG. In this case the contribution of the SNP nodes to the DAG penalty is not included in the score because it is the same for all models. We call this version 1 , and denote the score with the subscript 1 (score $\left._{\text {Epi1 }}\right)$. In the second version, only the $k$ SNPs that have edges to $D$ are included in the model and the remaining $n-k$ SNPs are excluded from the model. In this case, the contributions of the $k$ SNP nodes to the penalty are included because models with different values of $k$ have different penalties. We call this version 2 , and denote the score with the subscript 2 (e.g., score $_{E p i 2}$ ). The penalty term for $\operatorname{score}_{E p i}$ that is given in Equation 3 is for version 2.

After describing the results obtained using simulated data, we show those for real data.

\section{Simulated Data Results}

We evaluated the scoring criteria using simulated data sets that were developed from 70 genetic models with different heritabilities, minor allele frequencies and penetrance values. Each model consists of a probabilistic relationship in which 2 SNPs combined are correlated with the disease, but neither SNP is individually correlated. Each data set has sample size equal to $200,400,800$, or 1600 , and there are 7000 data sets of each size. More details of the datasets are given in the Methods section.

For each of the simulated data sets, we scored all 1-SNP, 2-SNP, 3-SNP, and 4-SNP DDAGs. The total number of DDAGs scored for each data set was therefore 6195 . Since in a real setting we would not know the number of SNPs in the model generating the data, all models were treated equally in the learning process; that is, no preference was given to 2-SNP models.

We say that a method correctly learns the model generating the data if it scores the DDAG representing the generating model highest out of all 6195 models. Table 1 shows the number of times out of 7000 data sets that each BN scoring criterion correctly learned the generating model for each sample size. In this table, the scoring criteria are listed in descending order according to the total number of times the correct model was learned. Table 1 shows a number of interesting results. First, the AIC score performed reasonably well on small sample sizes, but its performances degraded at larger sample sizes. Unlike the other BN scores, the DAG penalty in the AIC score does not increase with the sample size. Second, the K2 score did not perform well, particularly at small sample sizes. However, the MML1 score, which can be interpreted as the K2 score with an added DAG penalty, performed much better. This indicates that the DAG penalty in the K2 score may be too small and the increased penalty assigned by the MML1 score is warranted. Third, MDR performed well overall but substantially worse than the best performing scores. Fourth, the best results were obtained with the BDeu score at moderate values of $\alpha$. However, the results were very poor for large values of $\alpha$, which assign very small DAG penalties. 
Table 1 Accuracies of scoring criteria

\begin{tabular}{|c|c|c|c|c|c|c|}
\hline & Scoring Criterion & 200 & 400 & 800 & 1600 & Total \\
\hline 1 & score $_{\alpha=15}$ & 4379 & 5426 & 6105 & 6614 & 22524 \\
\hline 2 & score $_{\alpha}=12$ & 4438 & 5421 & 6070 & 6590 & 22519 \\
\hline 3 & score $_{\alpha}=18$ & 4227 & 5389 & 6095 & 6625 & 22336 \\
\hline 4 & score $_{\alpha}=9$ & 4419 & 5349 & 5996 & 6546 & 22313 \\
\hline 5 & score $_{\alpha=21}$ & 3989 & 5286 & 6060 & 6602 & 21934 \\
\hline 6 & score $_{\alpha=6}$ & 4220 & 5165 & 5874 & 6442 & 21701 \\
\hline 7 & score $_{\text {MML1 }}$ & 4049 & 5111 & 5881 & 6463 & 21504 \\
\hline 8 & score $_{\alpha}=24$ & 3749 & 5156 & 5991 & 6562 & 21448 \\
\hline 9 & score $_{M D R}$ & 4112 & 4954 & 5555 & 5982 & 20603 \\
\hline 10 & score $_{\alpha}=3$ & 3839 & 4814 & 5629 & 6277 & 20559 \\
\hline 11 & score $_{\text {Epi2 }}$ & 3571 & 4791 & 5648 & 6297 & 20307 \\
\hline 12 & score $_{\alpha}=30$ & 3285 & 4779 & 5755 & 6415 & 20234 \\
\hline 13 & score $_{\text {MML2 }}$ & 3768 & 4914 & 5754 & 5780 & 20216 \\
\hline 14 & score $_{\text {Epi1 }}$ & 2344 & 5225 & 6065 & 6553 & 20187 \\
\hline 15 & score $_{\text {suz } 1}$ & 3489 & 4580 & 5521 & 6215 & 19805 \\
\hline 16 & score $_{\alpha}=36$ & 2810 & 4393 & 5464 & 6150 & 18817 \\
\hline 17 & score $_{\alpha}=42$ & 2310 & 4052 & 5158 & 5895 & 17415 \\
\hline 18 & score $_{k 2}$ & 1850 & 3475 & 5095 & 6116 & 16536 \\
\hline 19 & score $_{\text {Suz } 2}$ & 2245 & 3529 & 4684 & 5673 & 16131 \\
\hline 20 & score $_{\alpha}=54$ & 1651 & 3297 & 4492 & 5329 & 14769 \\
\hline 21 & score $_{\text {AICZ }}$ & 3364 & 3153 & 2812 & 2520 & 11847 \\
\hline 22 & score $_{\text {AIC } 1}$ & 2497 & 1967 & 1462 & 1126 & 7052 \\
\hline 23 & score $_{\alpha}=162$ & 26 & 476 & 1300 & 2046 & 3848 \\
\hline
\end{tabular}

The number of times out of 7000 data sets that each scoring criterion identified the correct model for sample sizes of $200,400,800$, and 1600 . The last column gives the total accuracy over all sample sizes. The scoring criteria are listed in descending order of total accuracy.

The ability of the highest ranking score (the BDeu score $_{\alpha=15}$ ) to identify the correct model was compared to that of the next six highest ranking scores using the McNemar chi-square test (see Table 2). In a fairly small interval around $\alpha=15$ there is not a significant difference in performance. However, as we move away from $\alpha=15$ the significance becomes dramatic, as is the significance relative to the highest scoring non-BDeu score ( score $\left._{M M L 1}\right)$.

BDeu scores with values of $\alpha$ in the range 12 - 18 performed significantly better than all other scores. If our goal is only to find a score that most often scores the correct model highest on low-dimensional simulated data sets like the ones analyzed here, then our results support the use of these BDeu scores. However, in practice, we are interested in the discovery of promising SNP-disease associations that may be investigated for biological plausibility. So perhaps more relevant than whether the correct model scores the highest is the recall of the correct model relative to the highest scoring model. The recall is given by:
Table 2 Statistical comparison of accuracies of scoring criteria

\begin{tabular}{ccc}
\hline & Scoring Criterion & $p$-value \\
\hline 1 & score $_{\alpha=15}$ & NA \\
\hline 2 & score $_{\alpha=12}$ & 0.996 \\
\hline 3 & score $_{\alpha=18}$ & 0.076 \\
\hline 4 & score $_{\alpha=9}$ & 0.046 \\
\hline 5 & score $_{\alpha=21}$ & $4.086 \times 10^{-8}$ \\
\hline 6 & score $_{\alpha=6}$ & $3.468 \times 10^{-14}$ \\
\hline 7 & score $_{\text {MML1 }}$ & $1.200 \times 10^{-20}$ \\
\hline
\end{tabular}

$P$-values obtained by comparing the accuracy of the highest ranking scoring criterion (score ${ }_{\alpha}=15$ ) with the next six highest ranking scoring criteria using the McNemar chi-square test. Each $p$-value is obtained by comparing the accuracies for 28,000 data sets.

$$
\operatorname{recall}(S, T)=\frac{\#(S \cap T)}{\#(S)},
$$

where $S$ is the set of SNPs in the correct model, $T$ is the set of SNPs in the highest scoring model, and \# returns the number of items in a set. The value of the recall is 0 if and only if the two sets do not intersect, while it is 1 if and only if all the SNPs in the correct model are in the highest scoring model. Therefore, recall is a measure of how well the SNPs in the correct model were are discovered. Recall does not measure, however, the extent to which the highest scoring model has additional SNPs that are not in the correct model (i.e., false positives).

Table 3 shows the recall for the various scoring criteria. The criteria are listed in descending order of total recall. Overall, these results are the reverse of those in Table 1. The BDeu scores with large values of $\alpha$ and the AIC scores appear at the top of the list. Part of the explanation for this is that these BDeu scores and AIC scores incorporate small DAG penalties, which results in larger models often scoring higher. A larger model has a greater chance of containing the two interacting SNPs. Not surprisingly score $_{S u z 1}$ and score $_{S u z 2}$, which have the largest DAG penalties of the MDL scores, appear at the bottom of the list. MDR again performed well but substantially worse than the best performing scores.

Perhaps the smaller DAG penalty is not the only reason that the BDeu scores with larger values of $\alpha$ performed best. It is possible that the BDeu scores with larger values of $\alpha$ can better detect the interacting SNPs than the BDeu scores with smaller values, but that the scores with larger values do poorly at scoring the correct model (the one with only the two interacting SNPs) highest because they too often pick a larger model containing those SNPs. To investigate this possibility, we investigated how well the scores discovered models 
Table 3 Recall for scoring criteria

\begin{tabular}{|c|c|c|c|c|c|c|}
\hline & Scoring Criterion & 200 & 400 & 800 & 1600 & Total \\
\hline 1 & score $_{\alpha}=162$ & 5259 & 6043 & 6566 & 6890 & 24758 \\
\hline 2 & score $_{A / C 2}$ & 5204 & 5969 & 6511 & 6849 & 24533 \\
\hline 3 & score $_{\text {AIC1 }}$ & 5186 & 5960 & 6481 & 6830 & 24457 \\
\hline 4 & score $_{\alpha}=54$ & 5223 & 5941 & 6473 & 6813 & 24450 \\
\hline 5 & score $_{k 2}$ & 5303 & 5962 & 6371 & 6747 & 24383 \\
\hline 6 & score $_{\alpha}=42$ & 5203 & 5902 & 6425 & 6794 & 24324 \\
\hline 7 & score $_{\alpha}=36$ & 5181 & 5866 & 6395 & 6768 & 24210 \\
\hline 8 & score $_{\alpha}=30$ & 5147 & 5816 & 6352 & 6754 & 24069 \\
\hline 9 & score $_{\alpha}=24$ & 5080 & 5767 & 6300 & 6725 & 23872 \\
\hline 10 & score $_{\alpha}=21$ & 5031 & 5733 & 6265 & 6704 & 23733 \\
\hline 11 & score $_{M D R}$ & 4870 & 5710 & 6324 & 6748 & 23652 \\
\hline 12 & score $_{\alpha}=18$ & 4973 & 5681 & 6230 & 6681 & 23565 \\
\hline 13 & score $_{\alpha}=15$ & 4902 & 5622 & 6183 & 6647 & 23354 \\
\hline 14 & score $_{\text {Epil }}$ & 4984 & 5529 & 6105 & 6575 & 23193 \\
\hline 15 & score $_{\alpha}=12$ & 4786 & 5531 & 6119 & 6605 & 23041 \\
\hline 16 & score $_{\alpha}=9$ & 4649 & 5416 & 6026 & 6547 & 22638 \\
\hline 17 & score $_{\alpha=6}$ & 4383 & 5219 & 5901 & 6453 & 21956 \\
\hline 18 & score $_{\text {MML1 }}$ & 4151 & 5159 & 5903 & 6473 & 21686 \\
\hline 19 & score $_{\text {MML2 }}$ & 3881 & 4969 & 5780 & 6412 & 21042 \\
\hline 20 & score $_{\text {Epi2 }}$ & 3895 & 4901 & 5715 & 6329 & 20840 \\
\hline 21 & score $_{\alpha}=3$ & 3953 & 4862 & 5652 & 6285 & 20752 \\
\hline 22 & score $_{\text {Suz } 1}$ & 3618 & 4696 & 5595 & 6251 & 20160 \\
\hline 23 & score $_{\text {Suz2 }}$ & 2500 & 3712 & 4811 & 5737 & 17760 \\
\hline
\end{tabular}

The sum of the recall for each scoring criterion over 7000 data sets for sample sizes of $200,400,800$, and 1600 . The last column gives the total recall over all sample sizes. The scoring criteria are listed in descending order of total recall.

55-59 (See Supplementary Table one to [10]). These models have the weakest broad-sense heritability (0.01) and a minor allele frequency of 0.2 , and are therefore the most difficult to detect.

Table 4 shows the number of times the correct hardto-detect model scored highest for a representative set of the scores. Table 5 shows the $p$-values obtained when the highest ranking score (BDeu score ${ }_{\alpha}={ }_{54}$ ) is compared to the next five highest ranking scores using the McNemar chi-square test. The BDeu score with large values of $\alpha$ performed significantly better than all other scores.

The BDeu scores with large $\alpha$ values discovered the difficult models best, though they perform poorly on the average when all models were considered. An explanation for this phenomenon is that these scores can indeed find interacting SNPs better than scores with smaller values of $\alpha$. However, when the interacting SNPs are fairly easy to identify, their larger DAG penalties makes it harder for them to identify the correct model relative to other scores. On the other hand, when the SNPs are hard to detect, their better detection capability more than compensates for their increased DAG
Table 4 Accuracies of scoring criteria on most difficult models

\begin{tabular}{ccccccc}
\hline & Scoring Criterion & $\mathbf{2 0 0}$ & $\mathbf{4 0 0}$ & $\mathbf{8 0 0}$ & $\mathbf{1 6 0 0}$ & Total \\
\hline 1 & score $_{\alpha=54}$ & 14 & 48 & 167 & 352 & 581 \\
\hline 2 & score $_{\alpha=162}$ & 1 & 21 & 146 & 355 & 563 \\
\hline 3 & score $_{\alpha=36}$ & 13 & 46 & 155 & 318 & 532 \\
\hline 4 & score $_{\alpha=21}$ & 12 & 43 & 106 & 289 & 450 \\
\hline 5 & score $_{\alpha=18}$ & 11 & 37 & 91 & 274 & 413 \\
\hline 6 & score $_{\text {MDR }}$ & 3 & 25 & 79 & 245 & 352 \\
\hline 7 & score $_{\alpha=12}$ & 7 & 25 & 65 & 215 & 312 \\
\hline 8 & score $_{\text {AIC2 }}$ & 16 & 33 & 80 & 138 & 267 \\
\hline 9 & score $_{\alpha=9}$ & 5 & 20 & 48 & 186 & 259 \\
\hline 10 & score $_{\text {Epi1 }}$ & 4 & 16 & 47 & 179 & 246 \\
\hline 11 & score $_{\text {MML1 }}$ & 2 & 7 & 23 & 140 & 172 \\
\hline 12 & score $_{\alpha=3}$ & 3 & 6 & 13 & 86 & 108 \\
\hline 13 & score $_{\text {Epi2 }}$ & 0 & 1 & 4 & 72 & 77 \\
\hline 14 & score $_{S \text { Suz } 1}$ & 0 & 1 & 2 & 41 & 44 \\
\hline
\end{tabular}

The number of times out of 500 that each scoring criterion correctly learned the correct model in the case of the most difficult models (55-59) for sample sizes of $200,400,800$, and 1600 . The last column gives the total accuracy over all sample sizes. The scoring criteria are listed in descending order of accuracy.

penalty. Additional file 1 provides an illustrative example of this phenomenon. We hypothesize therefore that $B D e u$ scores with larger values of $\alpha$ can better indentify interacting SNPs, even if they sometimes include extra SNPs in the highest scoring model.

\section{GWAS Data Results}

We evaluated the scoring criteria using a late onset Alzheimer's disease (LOAD) GWAS data set. LOAD is the most common form of dementia in the above 65-yearold age group. It is a progressive neurodegenerative disease that affects memory, thinking, and behavior. The only genetic risk factor for LOAD that has been consistently replicated involves the apolipoprotein E (APOE) gene. The $\varepsilon 4$ APOE genotype increases the risk of

Table 5 Statistical comparison of accuracies of scoring criteria on most difficult models

\begin{tabular}{cc}
\hline Scoring Criterion & $\boldsymbol{p}$-value \\
\hline score $_{\alpha=54}$ & NA \\
\hline score $_{\alpha=162}=0.610$ \\
\hline score $_{\alpha=36}=147$ \\
\hline score $_{\alpha=21}=1.870 \times 10^{-5}$ \\
\hline score $_{\alpha=18}$ & $1.080 \times 10^{-7}$ \\
\hline score $_{\text {MDR }}$ & $7.254 \times 10^{-14}$ \\
\hline
\end{tabular}

$P$-values obtained by comparing the accuracy of the highest ranking scoring criterion (score ${ }_{\alpha=15}$ ) with the next five highest ranking scoring criteria using the McNemar chi-square test. Each $p$-value is obtained by comparing the accuracies for 2,000 data sets generated by the hardest-to-detect models. 
development of LOAD, while the $\varepsilon 2$ genotype is believed to have a protective effect.

The LOAD GWAS data set that we analyzed was collected and analyzed by Rieman et al. [16]. The data set contains records on 1411 participants (861 had LOAD and 550 did not), and consists of data on 312,316 SNPs and one binary genetic attribute representing the apolipoprotein E (APOE) gene carrier status. The original investigators found that SNPs on the GRB-associated binding protein 2 (GAB2) gene interacted with the APOE gene to determine the risk of developing LOAD. More details of this dataset are given in the Methods section.

To analyze this Alzheimer GWAS data set, for a representative subset of the scores listed in Table 1 we did the following. We pre-processed the data set by scoring all models in which APOE and one of the 312,316 SNPs are each parents of the disease node
LOAD. The SNPs from the top 100 highest-scoring models were selected along with APOE. Using these 101 loci, we then scored all 1, 2, 3, and 4 parent models making a total of 4,254,726 models scored. We judged the effectiveness of each score according to how well it replicated the results obtained by the original investigators in [16] that the GAB2 gene is associated with LOAD. We did this by determining how many of the score's 25 highest-scoring models contained a GAB2 SNP. Table 6 shows the results. The number in each cell in Table 6 is the number of SNPs in the model, and the letter $\mathrm{G}$ appears to the right of that number if a GAB2 SNP appears in the model. The second to the last row in the table shows the total number of models in the top 25 that contain a GAB2 SNP. The last row in the table shows the total number of different GAB2 SNPs appearing in the top 25 models.

Table 6 Evaluation of scoring criteria concerning detection of GAB2 SNPs

\begin{tabular}{|c|c|c|c|c|c|c|c|c|c|c|c|c|}
\hline Rank & $\alpha=3$ & $\alpha=12$ & $\alpha=21$ & $\alpha=54$ & $\alpha=162$ & $\alpha=1000$ & K2 & MML1 & MDLn & Suz1 & Epi2 & MDR \\
\hline 1 & 4 & 4 & 4 & $4 \mathrm{G}$ & $4 \mathrm{G}$ & 4 & $4 \mathrm{G}$ & $4 \mathrm{G}$ & $4 G$ & 3 & $4 \mathrm{G}$ & 4 \\
\hline 2 & 4 & 4 & 4 & $4 \mathrm{G}$ & $4 \mathrm{G}$ & 4 & $4 \mathrm{G}$ & $4 \mathrm{G}$ & $4 G$ & $3 G$ & $4 \mathrm{G}$ & 4 \\
\hline 3 & 4 & 4 & $4 G$ & $4 \mathrm{G}$ & 4 & 4 & $4 G$ & $4 G$ & $4 G$ & $3 G$ & $4 G$ & 4 \\
\hline 4 & 4 & 4 & 4 & $4 G$ & $4 G$ & 4 & 4 & 4 & $4 G$ & 3 & $4 G$ & 4 \\
\hline 5 & 4 & 4 & 4 & 4 & 4 & 4 & 4 & 3 & $4 G$ & 3 & 3 & 4 \\
\hline 6 & 4 & 4 & 4 & 4 & $4 \mathrm{G}$ & 4 & 4 & 4 & 4 & $3 G$ & $4 \mathrm{G}$ & $4 \mathrm{G}$ \\
\hline 7 & 4 & 4 & 4 & $4 G$ & 4 & 4 & 4 & 4 & 4 & $3 G$ & 4 & 4 \\
\hline 8 & 4 & 4 & 4 & 4 & 4 & 4 & 4 & 4 & $4 G$ & $3 G$ & 4 & $4 G$ \\
\hline 9 & 4 & 4 & 4 & $4 \mathrm{G}$ & 4 & 4 & 4 & 4 & $4 G$ & $3 G$ & $4 \mathrm{G}$ & 4 \\
\hline 10 & 4 & 4 & 4 & $4 \mathrm{G}$ & $4 \mathrm{G}$ & 4 & 4 & $3 G$ & 4 & 2 & $4 G$ & $4 \mathrm{G}$ \\
\hline 11 & 4 & 4 & $4 G$ & $4 G$ & $4 \mathrm{G}$ & $4 \mathrm{G}$ & $4 \mathrm{G}$ & 4 & 4 & 3 & 4 & 4 \\
\hline 12 & 4 & $4 \mathrm{G}$ & $4 \mathrm{G}$ & 4 & $4 \mathrm{G}$ & $4 G$ & $4 \mathrm{G}$ & 4 & 4 & $3 G$ & 4 & $4 \mathrm{G}$ \\
\hline 13 & 4 & 4 & 4 & $4 G$ & $4 \mathrm{G}$ & $4 \mathrm{G}$ & 4 & $4 G$ & $4 G$ & $3 G$ & 4 & 4 \\
\hline 14 & 4 & 4 & 4 & 4 & $4 G$ & 4 & $4 G$ & $4 G$ & 4 & 3 & $3 G$ & 4 \\
\hline 15 & 4 & 4 & $4 \mathrm{G}$ & $4 G$ & $4 \mathrm{G}$ & 4 & $4 \mathrm{G}$ & $3 G$ & 4 & $3 G$ & $4 G$ & $4 \mathrm{G}$ \\
\hline 16 & 4 & 4 & $4 \mathrm{G}$ & $4 G$ & $4 \mathrm{G}$ & $4 \mathrm{G}$ & $4 \mathrm{G}$ & 4 & 4 & $3 G$ & $3 G$ & 4 \\
\hline 17 & 4 & 4 & 4 & $4 G$ & $4 G$ & 4 & $4 G$ & 3 & $4 G$ & 3 & 4 & $4 G$ \\
\hline 18 & 4 & 4 & $4 \mathrm{G}$ & $4 \mathrm{G}$ & $4 \mathrm{G}$ & $4 G$ & 4 & $4 G$ & $4 G$ & $3 G$ & 4 & 4 \\
\hline 19 & 4 & 4 & $4 G$ & $4 G$ & $4 G$ & $4 G$ & 4 & $4 G$ & $4 G$ & $3 G$ & 4 & 4 \\
\hline 20 & 4 & 4 & 4 & $4 G$ & $4 G$ & $4 G$ & 4 & $4 G$ & 4 & $3 G$ & $4 G$ & $4 G$ \\
\hline 21 & 4 & 4 & 4 & $4 G$ & 4 & $4 G$ & 4 & $4 \mathrm{G}$ & $4 G$ & $3 G$ & $4 G$ & $4 G$ \\
\hline 22 & 4 & $4 G$ & 4 & $4 G$ & $4 \mathrm{G}$ & $4 \mathrm{G}$ & 4 & 4 & $4 G$ & 3 & $4 \mathrm{G}$ & 4 \\
\hline 23 & 4 & $4 \mathrm{G}$ & 4 & $4 G$ & 4 & $4 G$ & $4 G$ & 4 & $4 \mathrm{G}$ & $3 G$ & 4 & 4 \\
\hline 24 & 4 & 4 & $4 \mathrm{G}$ & $4 G$ & $4 \mathrm{G}$ & 4 & 4 & 4 & $4 G$ & $3 G$ & $4 \mathrm{G}$ & 4 \\
\hline 25 & 4 & 4 & 4 & 4 & $4 G$ & 4 & 4 & 4 & $4 G$ & $3 G$ & 3 & 4 \\
\hline Total \# G G\#\#GGG & 0 & 3 & 7 & 19 & 18 & 10 & 10 & 11 & 16 & 17 & 14 & 8 \\
\hline \# Diff G & 0 & 2 & 3 & 7 & 6 & 4 & 4 & 4 & 8 & 8 & 8 & 6 \\
\hline
\end{tabular}

Information about the 25 highest scoring models for a variety of scoring criteria. The number on the left in a cell is the number of SNPs in the model, and the letter G appears to the right of that number if a GAB2 SNP appears in the model. The second to the last row shows the total number of models in the top 25 that contained a GAB2 SNP. The last row shows the total number of different GAB2 SNPs appearing in the top 25 models. 
We included two new scores in this analysis. The first score is the BDeu score with $\alpha=1000$. We did this to test whether we can get good recall with arbitrarily high values of $\alpha$. The second new score is an MDL score with no DAG penalty (labelled MDLn in the table). We did this to investigate the recall for the MDL score when we constrain the highest scoring model to be one containing four parent loci.

These results substantiate our hypothesis that larger values of $\alpha$ (54 and 162) can better detect the interacting SNPs. For each of the BDeu scores, the 25 highestscoring models each contain 4 parent loci. However, when $\alpha$ equals 54 or 162,19 and 18 respectively of the 25 highest-scoring models contain a GAB2 SNP, whereas for $\alpha$ equal to 12 only 7 of them contain a GAB2 SNP, and for $\alpha$ equal to 3 none of them do. The results for $\alpha$ equal to 1000 are not very good, indicating that we cannot obtain good results for arbitrarily large values of $\alpha$. The MDL scores (MDLn, Suz1 and Epi2) all performed well, with the Suz1 score never selecting a model with more than 3 parent loci. This result indicates that the larger DAG penalty seems to have helped us hone in on the interacting SNPs. All the MDL scores detected the highest number of different GAB2 SNPs, namely 8 . In comparison, MDR did not perform very well, having only 8 models of the top 25 containing GAB2 SNPs and none of the top 5 containing GAB2 SNPs.

\section{Discussion}

We compared the performance of a number of $\mathrm{BN}$ scoring criteria when identifying interacting SNPs from simulated genetic data sets. Each data set contained 20 SNPs with two interacting SNPs and was generated from one of 70 different epistasis models. Jiang et al. [52] analyzed these same data sets using the BNMBL method and MDR (both of these methods are discussed in the Background section). However, that paper only investigated models with two interacting SNPs. So the 1-SNP, 3-SNP, and 4-SNP models were not competing and the learned model was restricted to be a 2-SNP model. In real applications we rarely would know how many SNPs are interacting. So this type of analysis is not as realistic as the one reported here.

Table 1 shows that the BDeu score with values of $\alpha$ between 12 and 18 was best at learning the correct model over all 28,000 simulated data sets. However, Table 3 shows that the BDeu score with large values of $\alpha$ (54 and 162) performed better at recall over all 28,000 data sets. Table 4 shows that these large values of $\alpha$ yield better detection of the models that are hardest to detect.

We evaluated the performance of a subset of the BN scores used in the simulated data analysis on a LOAD GWAS data set. The effectiveness of each score was judged according to how well it substantiated the previously obtained result that the GAB2 gene is associated with LOAD. As shown in Table 6, we obtained the best results with the BDeu score with large values of $\alpha$. The various MDL scores also performed well.

Overall, our results are mixed. Although scores with moderate values of $\alpha$ performed better at actually scoring the correct model highest using simulated data sets, scores with larger values of $\alpha$ performed better at recall, at detecting models that are hardest to detect, and at substantiating previous results using a real data set. Our main goal is to develop a method that can discover SNPs associated with a disease from real data. Therefore, based on the results reported here, it seems that it is more promising to use the BDeu score with large values of $\alpha$ (54-162), rather than smaller values.

The MDL scores also performed well in the case of the real data set. An explanation for their poor performance with the simulated data sets is that their DAG penalties are either too large or too small. If we simply used an MDL score with no DAG penalty we should be able to discover interacting SNPs well (as indicated by Table 6). Once we determine candidate interactions using these scores, we can perform further data analysis of the interactions and also investigate the biological plausibility of the genotype-phenotype relationships. However, additional research is needed to further investigate a DAG penalty appropriate to this domain.

Another consideration which was not investigated here is the possible increase in false positives with increased detection capability. That is, although the BDeu score with large values of $\alpha$ performed best at recall and at identifying hard-to-detect models, perhaps these scores may also score some incorrect models higher, and at a given threshold might have more false positives. Further research is needed to investigate this matter.

Additional file 1 provides an illustrative example and some theoretical justification as to why a BDeu score with large values of $\alpha$ should perform well at discovering hard-to-detect SNP-phenotype relationships. However, further research, both of a theoretical and empirical nature, is needed to investigate the pattern of results reported here. In particular, additional simulated data sets containing data on a large number of SNPs (numbers appearing in real studies) should be analyzed to see if the BDeu score with large values of $\alpha$ or some other approach performs better in this more realistic setting.

\section{Conclusions}

Our results indicate that representing epistatic interactions using $\mathrm{BNs}$ and scoring them using a $\mathrm{BN}$ scoring 
criteria holds promise for identifying epistatic relationships. Furthermore, they show that the use of the BDeu score with large values of $\alpha$ (54-162) can yield the best results on some data sets. Compared to MDR and other $\mathrm{BN}$ scoring criteria, these BDeu scores performed substantially better at detecting the hardest-to-detect models using simulated data sets, and at confirming previous results using a real GWAS data set.

\section{Methods}

\section{Simulated Data Sets}

Each simulated data set was developed from one of 70 epistasis models described in Velez et al. [10] (see Supplementary Table one in [10] for details of the 70 models). These datasets are available at http://discovery. dartmouth.edu/epistatic_data/.

Each model represents a probabilistic relationship in which two SNPs together are correlated with the disease, but neither SNP is individually predictive of disease. The relationships represent various degrees of penetrance, heritability, and minor allele frequency. The models are distributed uniformly among seven broadsense heritabilities ranging from 0.01 to 0.40 ( 0.01 , $0.025,0.05,0.10,0.20,0.30$, and 0.40$)$ and two minor allele frequencies (0.2 and 0.4).

Data sets were generated with case-control ratio (ratio of individuals with the disease to those without the disease) of 1:1. To create one data set they fixed the model. Based on the model, they then generated data concerning the two SNPs that were related to the disease in the model, 18 other unrelated SNPs, and the disease. For each of the 70 models, 100 data sets were generated for a total of 7000 data sets. This procedure was followed for data set sizes equal to 200, 400, 800 , and 1600.

\section{GWAS Data Set}

Several LOAD GWA studies have been conducted. We utilized data from one such study [16] that contains data on 312,316 SNPs. In this study, Reiman et al. investigated the association of SNPs separately in APOE $\varepsilon 4$ carriers and in APOE $\varepsilon 4$ noncarriers. A discovery cohort and two replication cohorts were used in the study. Within the discovery subgroup consisting of APOE $\varepsilon 4$ carriers, 10 of the 25 SNPs exhibiting the greatest association with LOAD (contingency test $p$-value $9 \times 10^{-8}$ to $1 \times 10^{-7}$ ) were located in the GRB-associated binding protein 2 (GAB2) gene on chromosome 11q14.1. Associations with LOAD for 6 of these SNPs were confirmed in the two replication cohorts. Combined data from all three cohorts exhibited significant association between LOAD and all 10 GAB2 SNPs. These 10 SNPs were not significantly associated with LOAD in the APOE $\varepsilon 4$ noncarriers.

\section{Implementation}

We implemented the methods for learning and scoring DDAGs using BN scoring criteria in the Java programming language. MDR v. mdr-2.0_beta_5 (available at http://www.epistasis.org) with its default settings (CrossValidation Count $=10$, Attribute Count Range $=1: 4$, Search Type $=$ Exhaustive) was used to run MDR. All experiments were run on a 32-bit Server running Windows 2003 with a $2.33 \mathrm{GHz}$ processor and $2.00 \mathrm{~GB}$ of RAM.

\section{Additional material}

Additional file 1: Illustrative Example of Better Large $\alpha$

Performance. This file provides an illustrative example to demonstrate a possible explanation for the better performance of the BDeu score at larger values of $\alpha$ on hard-to-detect genetic models.

\section{Acknowledgements}

The research reported here was funded in part by grant 1K99LM010822-01 from the National Library of Medicine.

\section{Author details}

${ }^{1}$ Department of Biomedical Informatics, University of Pittsburgh, Pittsburgh, PA, USA. ${ }^{2}$ Intelligent Systems Program, University of Pittsburgh, Pittsburgh, PA, USA. ${ }^{3}$ Clinical and Translational Science Institute, University Pittsburgh, Pittsburgh, PA, USA. ${ }^{4}$ Department of Human Genetics, University of Pittsburgh, Pittsburgh, PA, USA. ${ }^{5}$ Department of Computer Science, Northeastern Illinois University, Chicago, IL, USA.

\section{Authors' contributions}

XJ conceived the study, developed the DDAG model and the BNMBL score, conducted the experiments, and drafted the manuscript. RN identified the BN scores that were evaluated, performed the statistical analysis, and conceived and wrote Additional file 1. MB critically revised the manuscript for intellectual content concerning genetics. SV conceived the notion that we need not represent the relationships among SNPS, and critically revised the entire content of the manuscript. All authors read and approved the final manuscript.

Received: 21 July 2010 Accepted: 31 March 2011 Published: 31 March 2011

\section{References}

1. Bateson W: Mendel's Principles of Heredity New York; Cambridge University Press; 1909.

2. Moore $\mathrm{JH}$, Williams SM: New strategies for identifying gene gene interactions in hypertension. Annals of Medicine 2002, 34:88-95, 2002.

3. Ritchie $\mathrm{MD}$, et al: Multifactor-dimensionality reduction reveals high-order interactions among estrogen-metabolism genes in sporadic breast cancer. American Journal of Human Genetics 2001, 69:138-147.

4. Nagel Rl: Epistasis and the genetics of human diseases. $C R$ Biologies 2005, 328:606-615.

5. Armes BM, et al: The histologic phenotypes of breast carcinoma occurring before age 40 years in women with and without BRCA 1 or BRCA2 germline mutations. Cancer 2000, 83:2335-2345.

6. National Cancer Institute: Cancer Genomics. [http://www.cancer.gov/ cancertopics/understandingcancer/cancergenomics].

7. Heidema A, Boer J, Nagelkerke N, Mariman E, van der AD, Feskens E: The challenge for genetic epidemiologists: how to analyze large numbers of SNPs in relation to complex diseases. BMC Genetics 2006, 7:23, (21 April 2006).

8. Cho YM, Ritchie MD, Moore JH, Moon MK, et al: Multifactor dimensionality reduction reveals a two-locus interaction associated with type 2 diabetes mellitus. Diabetologia 2004, 47:549-554. 
9. Hahn LW, Ritchie MD, Moore JH: Multifactor dimensionality reduction software for detecting gene-gene and gene-environment interactions. Bioinformatics 2003, 19:376-382.

10. Velez $D R$, White $B C$, Motsinger AA, et al: A balanced accuracy function for epistasis modeling in imbalanced data sets using multifactor dimensionality reduction. Genetic Epidemiology 2007, 31:306-315.

11. Brookes AJ: The essence of SNPs. Gene 1999, 234:177-186.

12. Herbert A, Gerry NP, McQueen MB: A common genetic variant is associated with adult and childhood obesity. Journal of Computational Biology 2006, 312:279-384.

13. Spinola M, Meyer P, Kammerer S, et al: Association of the PDCD5 locus with long cancer risk and prognosis in Smokers. American Journal of Human Genetics 2001, 55:27-46.

14. Lambert JC, et al: Genome-wide association study identifies variants at CLU and CR1 associated with Alzheimer's disease. Nature Genetics 2009, 41:1094-1099.

15. Coon KD, et al: A high-density whole-genome association study reveals that APOE is the major susceptibility gene for sporadic late-onset Alzheimer's disease. Journal of Clinical Psychiatry 2007, 68:613-618.

16. Reiman EM, et al: GAB2 alleles modify Alzheimer's risk in APOE carriers. Neuron 2007, 54:713-720.

17. Brinza D, He J, Zelkovsky A: Optimization methods for genotype data analysis in epidemiological studies. In Bioinformatics Algorithms: Techniques and Applications. Edited by: Mandoiu I, Zelikovsky A. New York; Wiley; 2008:395-416.

18. Wu TT, Chen YF, Hastie T, Sobel E, Lange K: Genome-wide association analysis by lasso penalized logistic regression. Genome Analysis 2009, 25:714-721.

19. Wu J, Devlin B, Ringguist S, Trucco M, Roeder K: Screen and clean: A too for identifying interactions in genome-wide association studies. Genetic Epidemiology 2010, 34:275-285.

20. Wongseree $W$, et al: Detecting purely epistatic multi-locus interactions by an omnibus permutation test on ensembles of two-locus analyses. BMC Bioinformatics 2009, 10:294.

21. Zhang X, Pan F, Xie Y, Zou F, Wang W: COE: a general approach for efficient genome-wide two-locus epistasis test in disease association study. Journal of Computational Biology 2010, 17(3):401-415.

22. Meng Y, et al: Two-stage approach for identifying single-nucleotide polymorphisms associated with rheumatoid arthritis using random forests and Bayesian networks. BMC Proc 2007, 1(Suppl 1):S56

23. Wan $X$, et al: Predictive rule inference for epistatic interaction detection in genome-wide association studies. Bioinformatics 2010, 26(1):30-37.

24. Logsdon BA, Hoffman GE, Mezey JG: A variational Bayes algorithm for fast and accurate multiple locus genome-wide association analysis. BMC Bioinformatics 2010, 11:58.

25. Cordell HJ: Detecting gene-gene interactions that underlie human diseases. Nat Rev Genetics 2009, 10(6):392-404.

26. Thomas D: Methods for investigating gene-environment interactions in candidate pathway and genome-wide association studies. Annu Rev Public Health 2010, 31:1-8.

27. Castillo E, Gutiérrez JM, Hadi AS: Expert Systems and Probabilistic Network Models New York; Springer-Verlag; 2007.

28. Jensen FV: An Introduction to Bayesian Networks New York; Springer-Verlag; 1997.

29. Jensen FV, Neilsen TD: Bayesian Networks and Decision Graphs New York; Springer-Verlag; 2007

30. Korb K, Nicholson AE: Bayesian Artificial Intelligence Boca Raton, FL; Chapman \& Hall/CRC; 2003

31. Neapolitan RE: Probabilistic Reasoning in Expert Systems New York; Wiley; 1990.

32. Neapolitan RE: Learning Bayesian Networks Upper Saddle River, NJ; Prentice Hall; 2004

33. Pearl J: Probabilistic Reasoning in Intelligent Systems Burlington, MA; Morgan Kaufmann; 1988

34. Fishelson M, Geiger D: Exact genetic linkage computations for general pedigrees. Bioinformatics 2002, 18(Suppl 1):189-198.

35. Fishelson M, Geiger D: Optimizing exact genetic linkage computation. Journal of Computational Biology 2004, 11:263-275.

36. Friedman N, Koller K: Being Bayesian about network structure: a Bayesian approach to structure discovery in Bayesian networks. Machine Learning 2003, 20:95-126
37. Friedman N, Goldszmidt M, Wyner A: Data analysis with Bayesian networks: a bootstrap approach. In Proceedings of the Fifteenth Conference on Uncertainty in Artificial Intelligence. Edited by: Laskey KB, Prade H. Burlington, MA; Morgan Kaufmann; 1999:196-205.

38. Friedman N, Linial M, Nachman I, Pe'er D: Using Bayesian networks to analyze expression data. Proceedings of the Fourth Annual International Conference on Computational Molecular Biology 2005, 127-135.

39. Friedman N, Ninio M, Pe'er I, Pupko T: A structural EM algorithm for phylogenetic inference. Journal of Computational Biology 2002, 9(2):331-353

40. Neapolitan RE: Probabilistic Methods for Bioinformatics: with an Introduction to Bayesian networks Burlington, MA: Morgan Kaufmann; 2009.

41. Segal E, Pe'er D, Regev A, Koller D, Friedman N: Learning module networks. Journal of Machine Learning Research 2005, 6:557-588.

42. Spirtes P, Glymour C, Scheines R: Causation, Prediction, and Search. second edition. New York; Springer-Verlag; Boston, MA; MIT Press; 1993, 2000.

43. Perrier $E$, Imoto $S$, Miyano S: Finding optimal Bayesian network given a super-structure. Journal of Machine Learning Research 2008, 9:2251-2286.

44. Cooper GF, Herskovits E: A Bayesian method for the induction of probabilistic networks from data. Machine Learning 1992, 9:309-347.

45. Heckerman D, Geiger D, Chickering D: Learning Bayesian Networks: The Combination of Knowledge and Statistical Data. Technical Report MSRTR-94-09, Microsoft Research, Redmond, Washington; 1995.

46. Silander T, Kontkanen P, Myllymäki P: On sensitivity of the MAP Bayesian network structure to the equivalent sample size parameter. In Proceedings of the Twenty-Third Conference on Uncertainty in Artificial Intelligence. Edited by: Parr R, van der Gaag L. Corvallis, Oregon; AUAI Press; 2002:360-367.

47. Rissanen J: Modeling by shortest data description. Automatica 1978, 14:465-471.

48. Suzuki J: Learning Bayesian belief networks based on the minimum description length principle: basic properties. IEICE Transactions on Fundamentals 1999, E82-A:2237-2245.

49. Chickering M: Learning Bayesian networks is NP-complete. In Learning from Data: Lecture Notes in Statistics. Edited by: Fisher D, Lenz H. New York: Springer Verlag: 1996:121-130

50. Han B, Park M, Chen X: A Markov blanket-based method for detecting causal SNPs in GWAS. BMC Bioinformatics 2010, 11(Suppl 3):S5.

51. Verzilli CJ, Stallard N, Whittaker JC: Bayesian graphical models for genomewide association studies. The American Journal of Human Genetics 2006, 79:100-112.

52. Jiang $X$, Barmada MM, Visweswaran S: Identifying genetic interactions from genome-wide data using Bayesian networks. Genet Epidemiol 2010, 34(6):575-581

53. Visweswaran S, Wong Al, Barmada MM: A Bayesian method for identifying genetic interactions. Proceedings of the Fall Symposium of the American Medical Informatics Association 2009, 673-677.

54. Sebastiani P: Genetic dissection and prognostic modeling of overt stroke in sickle cell anemia. Nature Genetics 2005, 37:435-440.

55. Meng $Y$, et al: Two-stage approach for identifying single-nucleotide polymorphisms associated with rheumatoid arthritis using random forests and Bayesian networks. BMC Proc 2007, 1(Suppl 1):S56.

56. Robinson RW: Counting unlabeled acyclic digraphs. In Lecture Notes in Mathematics. Volume 622. Edited by: Little CHC. New York: Springer-Verlag; 1977:28-43.

57. Friedman N, Yakhini Z: On the sample complexity of learning Bayesian networks. Proceedings of the Twelfth Conference on Uncertainty in Artificial Intelligence 1996, 206-215.

doi:10.1186/1471-2105-12-89

Cite this article as: Jiang et al: Learning genetic epistasis using

Bayesian network scoring criteria. BMC Bioinformatics 2011 12:89. 